\title{
Combination Therapy With S-1, Oxaliplatin and Leucovorin in Patients With Advanced Esophageal Squamous Cell Carcinoma
}

\author{
NAOHIRO NISHIDA $^{1 *}$, MAKOTO YAMSAKI $^{1 *}$, KAZUKI ODAGIRI $^{1}$, KOTARO YAMASHITA $^{1}$, \\ KOJI TANAKA ${ }^{1}$, DAISUKE SAKAI ${ }^{2}$, TOMOKI MAKINO ${ }^{1}$, TSUYOSHI TAKAHASHI $^{1}$, \\ YUKINORI KUROKAWA ${ }^{1}$, TAROH SATOH ${ }^{2}$, MASAKI MORI ${ }^{3}$ and YUICHIRO DOKI ${ }^{1}$ \\ ${ }^{I}$ Department of Gastroenterological Surgery, Graduate School of Medicine, Osaka University, Suita, Japan; \\ ${ }^{2}$ Department of Frontier Science for Cancer and Chemotherapy, \\ Osaka University Graduate School of Medicine, Suita, Japan; \\ ${ }^{3}$ Department of Surgery and Science, Graduate School of Medical Sciences, Kyushu University, Fukuoka, Japan
}

\begin{abstract}
Background/Aim: In this study, we assessed the safety and efficacy of combination therapy with $S$-1, oxaliplatin and leucovorin (SOL) in advanced esophageal squamous cell carcinoma (ESCC) patients. Patients and Methods: Ten unresectable or recurrence ESCC patients, who had been previously treated with more than two regimens were included in this study. The treatment schedule comprised S-1 40-60 mg and fixed dose of leucovorin $25 \mathrm{mg}$ together orally twice a day for one week, followed by one-week of rest. Oxaliplatin 85 $\mathrm{mg} / \mathrm{m}^{2}$ was given as an intravenous infusion on day one, repeated every two weeks. Results: Of the eight patients with measurable lesions, two patients with partial response (25\%) and two with stable disease (25\%) were observed. Disease control rate was $50 \%$. Median progression-free survival and overall survival were 5.0 and 9.3 months, respectively. The main common adverse events were malaise (60\%), decreased appetite (50\%), peripheral sensory neuropathy (40\%). Conclusion: SOL therapy showed promising antitumor activity with acceptable toxicity even for heavily pretreated ESCC.
\end{abstract}

Esophageal cancer is the ninth most common cancer and the sixth leading cause of cancer-related deaths worldwide.

This article is freely accessible online.

*These Authors contributed equally to this work.

Correspondence to: Makoto Yamasaki, Department of Gastrointestinal Surgery, Graduate School of Medicine, Osaka University, 2-2 Yamadaoka, Suita, Osaka 565-0871, Japan. Tel: +81 0668792641, 2640, 3251, Fax: +81 0668792639, 3259, e-mail: myamasaki@gesurg.med.osaka-u.ac.jp

Key Words: Oxaliplatin, SOL, ESCC.
Squamous cell carcinoma is the most common histological type of esophageal cancer, which exhibits a high-incidence in eastern Asia including China and Japan (1). Although chemotherapy or radiation therapy (CRT) followed by extensive surgery has improved prognosis, unresectable or recurrent cases have a poor prognosis with a considerable decline in health-related quality of life (HRQoL).

Several chemotherapy regimens are in clinical use for unresectable or metastatic ESCC. Cisplatin (CDDP) and 5-fluorouracil (5-FU) based regimens has been a standard treatment for ESCC. In recent years, triplet regimens, cisplatin and fluorouracil plus docetaxel (DCF) have been regarded as a promising therapy, especially in neoadjuvant settings $(2,3)$. However, these regimens are nephrotoxic and emetogenic, therefore, sometimes too toxic for patients who have undergone multiple chemotherapy cycles. Patients with recurrent and metastatic esophageal cancer can easily become malnourished due to appetite loss and in some cases esophageal stricture (4). Furthermore, there are few second or later-line chemotherapy options, and response rate of these regimens are far from being satisfactory. New drug combination strategies should be considered for better prognosis of advanced ESCC.

$\mathrm{S}-1$, an oral prodrug of 5-FU-containing regimens are increasingly used for $\operatorname{ESCC}(3,5)$. Previous reports have shown the combination of S-1 with other key chemotherapeutic agents, including oxaliplatin, enhances the anti-tumor activity (6). Oxaliplatin, a platinum derivative, which has less nephrotoxic and emetogenic effects than the traditionally used CDDP, has been widely used in a variety of regimens for colorectal, pancreatic and gastric cancer (7). However, the anti-tumor activity of oxaliplatin and its combination with S-1 has not been sufficiently investigated in ESCC. A previous report demonstrated that SOL (S-1, 
oxaliplatin plus leucovorin) showed a high objective response rate $(66 \%)$ with acceptable toxic effects in advanced gastric cancer (8). In this study, we have investigated the efficiency and safety of SOL therapy in patients with advanced ESCC, who have been pretreated with various chemotherapeutic agents and/or CRT.

\section{Patients and Methods}

Patients. Between January 2017 and February 2018, 10 patients with histologically proven squamous cell carcinoma of the thoracic esophagus, who experienced recurrence after curative surgery or underwent $\mathrm{R} 2$ resection were included in this study. All patients were mainly treated at the Osaka University hospital. All patients had been previously treated with at least two regimens of chemotherapy. Patients with an Eastern Cooperative Oncology Group performance status of $0-1$, and with no serious vital organ dysfunction (hematologic, liver, and renal) were selected based on the following values: platelet count $\geq 10 \times 10^{4} / \mathrm{mm}^{3}$, hemoglobin $\geq 8.0 \mathrm{~g} / \mathrm{dl}$, neutrophil count $\geq 1,500 / \mathrm{mm}^{3}$, serum bilirubin $\leq 1.5 \mathrm{mg} / \mathrm{dl}$, serum aspartate aminotransferase and alanine aminotransferase $\leq$ twice the upper limit of normal range, and creatinine $\leq 1.2 \mathrm{mg} / \mathrm{dl}$. Reduced S-1 dose was used for patients with estimated Glomerular filtration rate (eGFR) $<60$. Pathological stage was evaluated according to the seventh edition of the Tumor Node Metastasis Classification of the International Union Against Cancer (UICC-TNM). This study was approved by medical review committee of Osaka University School of Medicine and informed consent was obtained from all patients.

Study treatment. The SOL regimen comprised 40-60 mg of S-1 and a fixed dose of $25 \mathrm{mg}$ of leucovorin, administered orally, twice a day for one week, followed by one-week of rest. Oxaliplatin, $85 \mathrm{mg} / \mathrm{m}^{2}$, was given as an intravenous infusion on day 1, and repeated every 2 weeks (Figure 1). Dose reduction was set as follows; $85 \mathrm{mg} / \mathrm{m}^{2}$, $65 \mathrm{mg} / \mathrm{m}^{2}$, and $50 \mathrm{mg} / \mathrm{m}^{2}$ for oxaliplatin, $60 \mathrm{mg}, 50 \mathrm{mg}$, and $40 \mathrm{mg}$ twice daily for $\mathrm{S}-1$. If grade 3 or worse gastrointestinal disorders including nausea, diarrhea or stomatitis were observed, the dose of S-1 was reduced. If grade 3 or worse peripheral sensory neuropathy was observed, oxaliplatin was withheld until neuropathy improved to grade 2 or better, and the dose of oxaliplatin was reduced in the following treatment. If grade 3 or worse neutropenia or thrombocytopenia, or febrile neutropenia was recorded, the dose of all drugs except for leucovorin was reduced. Treatment was continued until disease progression, the development of serious adverse effect, or the patient refused further treatment.

Outcomes. The primary end point was overall response rate (ORR). Secondary end points were overall survival (OS), progression-free survival (PFS) and safety. Tumor response was assessed using computed tomography (CT) scans of the chest and abdomen within six weeks before the start of the treatment and were repeated every 12 weeks until discontinuation of protocol treatment. Results were evaluated by RECIST v1.1 for response rate and disease control rate. Change in diameter of target lesions from baseline was assessed at the time of best response. The severity of adverse events during the treatment period was evaluated according to the National Cancer Institute Common Terminology Criteria for Adverse Events (version 4.0). Safety and laboratory data were assessed at baseline and at least once every two weeks after the start of treatment.

Day $1 \quad$ Day $8 \quad$ Day 15

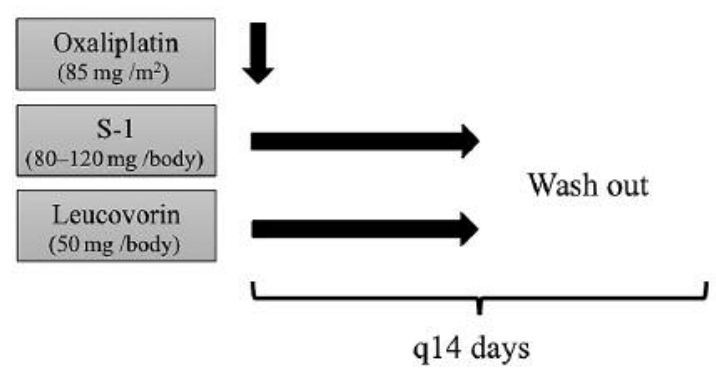

Figure 1. Schedule of study treatment.

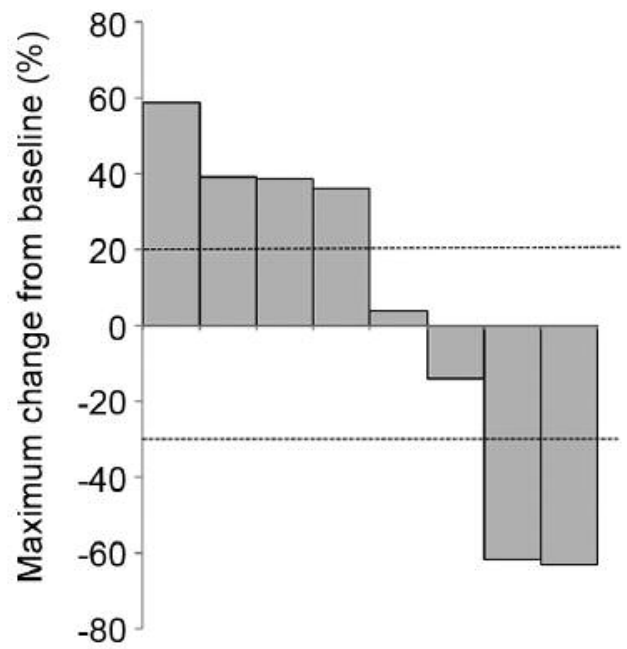

Patients

Figure 2. Change in size of target lesions from baseline, analyzed in patients with measurable disease $(n=8)$.

Statistical analysis. OS and PFS were calculated by the KaplanMeier method. OS was defined as the time from the first induction of SOL therapy to death from any cause, or the date of last contact in the case of continuing survival. PFS was defined as the time from the first induction of SOL therapy to disease progression or death from any cause.

\section{Results}

Patient characteristics. The baseline characteristics of patients are shown in Table I. Eight patients experienced recurrence after curative surgery and two patients underwent R2 resection. All patients were treated with at least two regimens of chemotherapy and were refractory or intolerant to fluoropyrimidine-based, platinum-based, and taxane-based chemotherapy. Four patients had received CRT before SOL 


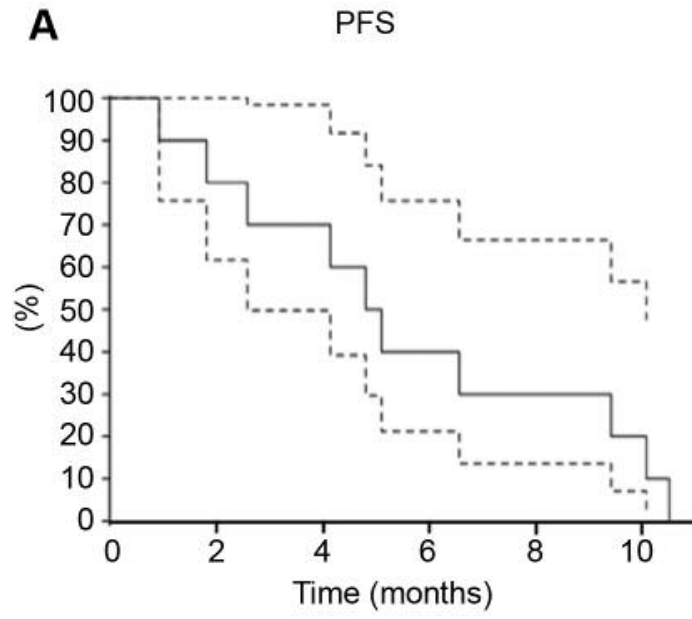

Median PFS 5.0 months (95\% Cl 2.6-NA)

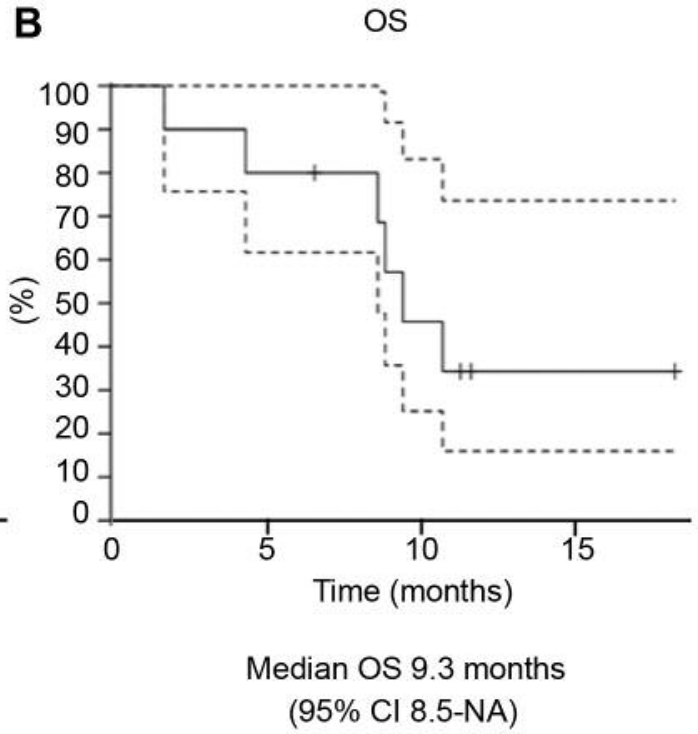

Figure 3. Kaplan-Meier analysis of PFS (A) and OS (B). Dashed line indicates $95 \%$ confidence interval.

therapy. The most commonly used regimens were paclitaxel treatment (8/10) and DCF with or without radiotherapy (8/10). Cisplatin plus 5-FU (FP) was administered in 4 patients. Of the eight patients who experienced recurrence after R0 resection, six patients had lymph node (mediastinal, cervical and abdominal lymph nodes) recurrence. Liver metastasis was observed in two patients, and lung, peritoneal, and adrenal gland metastasis was observed in one patient each.

Treatment and compliance. Patients received a median of 9.5 cycles (range=5.8-10.8 cycles) of SOL therapy. Seven patients $(70 \%)$ required dose reduction of oxaliplatin according to adverse events including peripheral sensory neuropathy, decreased appetite and malaise. Seven patients (70\%) required dose reduction of S-1 according to stomatitis, decreased appetite and malaise.

Tumor response. Eight of the ten patients had measurable disease and were evaluated by RECIST1.1 criteria. Partial response (PR) and stable disease (SD) were observed in $25 \%$ $(\mathrm{n}=2)$ and $25 \%(\mathrm{n}=2)$, respectively. ORR was $25 \%$ and disease control rate was $50 \%$ (Table II). For these patients with measurable lesions, changes in the size of target lesions from baseline were calculated and shown in Figure 2. In four patients with lung metastasis, ORR was $25 \%$ [PR $n=1 / \mathrm{SD}$ $n=1 /$ progressive disease (PD) $n=2$ ]. Two cases of liver metastasis demonstrated one PR and one PD.

$O S$ and RFS. At the time of analysis, median follow-up was 9.1 months (range=7.0-11.0 months). All patients experienced
PD and six people died during the follow-up period. Median OS and PFS were 5.0 months $(95 \% \mathrm{CI}=2.6-\mathrm{NA})$ and 9.3 months $(95 \% \mathrm{CI}=8.5-\mathrm{NA})$, respectively (Figure 3$)$.

Toxicity. Frequently observed non-haematological toxicities included malaise (G1/2 60\%; G3/40\%), decreased appetite (G1/2 40\%; G3/4 10\%) and fatigue (G2 40\%). Peripheral neuropathy was observed in $40 \%(\mathrm{G} 1 / 240 \%)$ of the patients, although a G3 event was not observed in this study (Table III). Bone marrow suppression was relatively mild, with $10 \%(n=1)$ of the patients experiencing G4 leucopenia, 20\% ( $n=2)$ had G3 neutropenia and $10 \%(\mathrm{n}=1)$ had G3 thrombocytopenia. Anemia (G1/2 90\%; G3 10\%) was frequently observed in this study.

\section{Discussion}

Growing evidence has been accumulated regarding chemotherapy and CRT for ESCC. The standard FP regimen showed an ORR of $35 \%(9,10)$, while the recently developed triplet regimen, DCF, demonstrated an ORR of $60-75 \%$ (11-13). OS improvement from the DCF regimen is currently being investigated in a phase III trial. Taxanes are also frequently used in second line therapy, and paclitaxel has shown an ORR of 20-44\% (14-16). However, most of the evidence so far has been limited to first- and second-line treatments, and later line chemotherapy for advanced ESCC has not been intensively explored.

In this retrospective study, we have demonstrated that SOL therapy is effective in heavily treated advanced ESCC patients with a manageable tolerability profile. Treatment of 
Table I. Baseline characteristics.

\begin{tabular}{lc}
\hline Gender & \\
Male & $10(100 \%)$ \\
Female & $0(0 \%)$ \\
Median age (range) & $62(43-75)$ \\
ECOG performance status & \\
0 & $6(60 \%)$ \\
1 & $4(40 \%)$ \\
Histological type & $10(100 \%)$ \\
Squamous cell carcinoma & $0(0 \%)$ \\
Others & \\
Tumor status & $2(20 \%)$ \\
Unrespectable & $8(80 \%)$ \\
Recurrence & $10(100 \%)$ \\
Previous treatment & $4(40 \%)$ \\
Surgery & $10(100 \%)$ \\
Radiotherapy & $6(60 \%)$ \\
Chemotherapy & $3(30 \%)$ \\
2 regimens & $1(10 \%)$ \\
3 regimens & \\
4 regimens & $7(70 \%)$ \\
Number of organs with metastases & $2(20 \%)$ \\
1 & $1(10 \%)$ \\
2 &
\end{tabular}

patients with metastatic and recurrent esophageal cancer needs to be carefully considered, because of deterioration of the general condition due to malnutrition or prolonged adverse events caused by previously administered chemotherapy (17). SOL can be administered on an outpatient basis every two weeks, and our results showed that this regimen is effective and well-tolerated in patients who have been previously administered two or more chemotherapy regimens.

FP regimen has been regarded as standard treatment for esophageal cancer; however, recent reports have demonstrated that oxaliplatin is as effective as cisplatin in patients with esophagogastric cancer (18). Combination of 5-FU, leucovorin and oxaliplatin (FLO) was shown to be less toxic than 5-FU/leucovorin/ cisplatin (FLP) in a phase III trial of gastric and gastroesophageal cancers (19). The combination of oxaliplatin, capecitabine and oral fluoropyrimidine has also been shown to be effective in advanced or metastatic esophageal cancer (20). Intensive infusion and hospitalization are not required for regimens including oxaliplatin, making this drug a useful chemotherapy option for gastrointestinal cancers. Although pre-clinical experimental models have shown that there is possibly cross-resistance of oxaliplatin and cisplatin (21), a previous phase II study of oxaliplatin, 5-FU and LV demonstrated efficacy in previously cisplatin-treated gastric cancer patients (22). Because all patients recruited in this study had received cisplatin-containing regimens before SOL
Table II. Best overall response.

\begin{tabular}{lll}
\hline & $\mathrm{n}=8$ & \\
\hline Complete response & 0 & \\
Partial response & 2 & $25 \%$ \\
Stable disease & 2 & $25 \%$ \\
Progressive disease & 4 & $50 \%$ \\
Not evaluable & 0 & \\
Overall response rate* $^{*}$ & 2 & $25 \%$ \\
Disease control rate $^{\dagger}$ & 4 & $50 \%$ \\
\hline
\end{tabular}

*Complete or partial response. ${ }^{\dagger}$ Complete response, partial response, or stable disease.

therapy, use of this treatment in an early line setting might be beneficial. In fact, SOL regimen was used as first-line treatment for advanced gastric cancer and showed a promising ORR in a previous study (8).

Leucovorin facilitates the anti-tumor effect of fluorouracil through enhancing the inhibition of thymidylate synthase activity. Hironaka et al. demonstrated that objective response rate was similar between $\mathrm{S} 1$ plus leucovorin and $\mathrm{S} 1$ plus CDDP (43\% vs. $46 \%, p=0.84$ ), and that addition of SOL showed a much better objective response rate $(66 \%)$ in advanced gastric cancer (8). Although most of the evidence obtained so far is in regard to adenocarcinoma of the esophagus, previous reports have suggested that oxaliplatincontaining regimens are also effective in ESCC (23). FOLFOX6 has been shown to be effective as a first-line treatment of ESCC. The overall response rate and disease control rate were $23.2 \%$ and $67.9 \%$, respectively. The median PFS was 4.4 months, and the median OS was 7.7 months (24). Safety and efficacy of SOL therapy in ESCC have been largely unknown, especially in later-line treatments. In this study, 2 (20\%) showed PR and $2(20 \%)$ showed SD to oxaliplatin-based SOL treatment, despite heavy pre-treatment with cisplatin-containing regimens. This might be because SOL regimen is well-tolerated and a relatively high dose intensity of oxaliplatin is maintained during a median treatment period of 5.0 months (3.0-8.8).

In regard to the toxicity, peripheral neuropathy was commonly observed in $40 \%$ of the patients, although a G3 event was not observed in this study. Because 8 to 10 patients had received paclitaxel or paclitaxel-containing regimens before SOL, the influence of prior treatment must be considered. A previous study in gastric cancer also showed a relatively high frequency of G1/2 neuropathy events and $9 \%$ of $\mathrm{G} 3$ events (8). Bone marrow suppression was relatively mild, with $10 \% \quad(n=1)$ of the patients experiencing G4 leucopenia, 20\% $(n=2)$ had G3 neutropenia and $10 \%(\mathrm{n}=1)$ had G3 thrombocytopenia. Anemia $(\mathrm{G} 1 / 2$ 90\%; G3 10\%) was more commonly observed, although moderate anemia has already been observed at the start of 
Table III. Adverse events.

\begin{tabular}{|c|c|c|c|c|c|c|}
\hline & G1 & $\mathrm{G} 2$ & G3 & G4 & Any Grade & $\geq$ Grade 3 \\
\hline \multicolumn{7}{|c|}{ Non-haematological adverse events } \\
\hline Stomatitis & $1(10 \%)$ & 0 & $2(20 \%)$ & 0 & $3(30 \%)$ & $2(20 \%)$ \\
\hline Diarrhea & $3(30 \%)$ & 0 & 0 & 0 & $3(30 \%)$ & 0 \\
\hline Nausea & $3(30 \%)$ & 0 & 0 & 0 & $3(30 \%)$ & 0 \\
\hline Fatigue & 0 & $4(40 \%)$ & 0 & 0 & $4(40 \%)$ & 0 \\
\hline Malaise & 0 & $6(60 \%)$ & 0 & 0 & $6(60 \%)$ & 0 \\
\hline Decreased appetite & $2(20 \%)$ & $2(20 \%)$ & $1(10 \%)$ & 0 & $5(50 \%)$ & $1(10 \%)$ \\
\hline Peripheral sensory neuropathy & $1(10 \%)$ & $3(30 \%)$ & 0 & 0 & $4(40 \%)$ & 0 \\
\hline Creatinine increased & 0 & 0 & 0 & 0 & 0 & 0 \\
\hline AST/ALT increased & 0 & 0 & 0 & 0 & 0 & 0 \\
\hline \multicolumn{7}{|l|}{ Haematological adverse events } \\
\hline Leucopenia & $1(10 \%)$ & 0 & 0 & $1(10 \%)$ & $2(20 \%)$ & $1(10 \%)$ \\
\hline Neutropenia & 0 & $1(10 \%)$ & $2(20 \%)$ & 0 & $3(30 \%)$ & $2(20 \%)$ \\
\hline Anemia & $3(30 \%)$ & $6(60 \%)$ & 0 & $1(10 \%)$ & $10(100 \%)$ & $1(10 \%)$ \\
\hline Thrombocytopenia & $4(40 \%)$ & 0 & $1(10 \%)$ & 0 & $5(50 \%)$ & $1(10 \%)$ \\
\hline Febrile neutropenia & 0 & 0 & $1(10 \%)$ & 0 & $1(10 \%)$ & $1(10 \%)$ \\
\hline
\end{tabular}

treatment in most of the patients, due to the long history of prior chemotherapy. The frequency of neutropenia was relatively low compared with previous studies showing the effectiveness of SOL in gastric cancer, where $26 \%$ of patients in the SOL cohort showed G3/4 neutropenia (8).

The limitations of this study were the small sample size, a wide variety of prior treatment regimens including CRT and a relatively short follow-up duration. Nevertheless, the efficacy and safety data of the SOL regimen shown here might be useful information for heavily pretreated ESCC patients, who have few treatment options available.

\section{Conflicts of Interest}

N. Nishida: Yakult Honsha Co., Ltd., Chugai Pharmaceutical Co., Ltd., and Ono Pharmaceutical Co., Ltd. T. Satoh: Yakult Honsha Co., Ltd., Chugai Pharmaceutical Co., Ltd., and Ono Pharmaceutical Co., Ltd. D. Sakai: Yakult Honsha Co., Ltd., Chugai Pharmaceutical Co., Ltd., and Ono Pharmaceutical Co., Ltd. All other Authors have declared no conflicts of interest regarding this study.

\section{Authors' Contributions}

MY initiated this project. NN, MY, KO, KY, KT, DS, TM, TT, YK, $\mathrm{TS}, \mathrm{MM}$ and $\mathrm{YD}$ designed the study protocol and wrote the manuscript. KO, NN and MY collected clinical information and performed statistical analysis.

\section{Acknowledgements}

Institutional endowments were received partially from Chugai Co., Ltd., Yakult Honsha Co., Ltd. and Ono Pharmaceutic Co., Ltd. These funding sources had no role in the main experimental equipment, supply expenses, study design, data collection and analysis, decision to publish, or preparation of the manuscript. The Authors thank T. Ishikawa and Y. Shimatani for their technical assistance.

\section{References}

1 Lagergren J, Smyth E, Cunningham D and Lagergren P: Oesophageal cancer. Lancet 390: 2383-2396, 2017. PMID: 21112905. DOI: 10.1016/S0140-6736(17)31462-9

2 Yamasaki M, Yasuda T, Yano M, Hirao M, Kobayashi K, Fujitani K, Tamura S, Kimura Y, Miyata H, Motoori M, Shiraishi O, Makino T, Satoh T, Mori M and Doki Y: Multicenter randomized phase II study of cisplatin and fluorouracil plus docetaxel (DCF) compared with cisplatin and fluorouracil plus Adriamycin (ACF) as preoperative chemotherapy for resectable esophageal squamous cell carcinoma (OGSG1003). Ann Oncol 28: 116-120, 2017. PMID: 27687307. DOI: 10.1093/annonc/mdw439

3 Tanaka Y, Yoshida K, Suetsugu T, Imai T, Matsuhashi N and Yamaguchi K: Recent advancements in esophageal cancer treatment in Japan. Ann Gastroenterol Surg 2: 253-265, 2018. DOI: $10.1002 /$ ags 3.12174

4 Kato K, Muro K, Ando N, Nishimaki T, Ohtsu A, Aogi K, Aoyama N, Nagai K and Kato H: A phase II study of nedaplatin and 5-fluorouracil in metastatic squamous cell carcinoma of the esophagus: The Japan Clinical Oncology Group (JCOG) Trial (JCOG 9905-DI). Esophagus 11: 183-188, 2014. DOI: 10.1007/s 10388-014-0427-7

5 Tanaka Y, Yoshida K, Tanahashi T, Okumura N, Matsuhashi N and Yamaguchi K: Phase II trial of neoadjuvant chemotherapy with docetaxel, nedaplatin, and S1 for advanced esophageal squamous cell carcinoma. Cancer Sci 107: 764-772, 2016. PMID: 27061001. DOI: 10.1111/cas.12943

6 Yamada Y, Higuchi K, Nishikawa K, Gotoh M, Fuse N, Sugimoto N, Nishina T, Amagai K, Chin K, Niwa Y, Tsuji A, Imamura $H$, Tsuda M, Yasui H, Fujii H, Yamaguchi K, Hironaka S, Shimada K, Miwa H, Hamada C and Hyodo I: Phase III study comparing oxaliplatin plus S-1 with cisplatin plus S-1 in 
chemotherapy-naïve patients with advanced gastric cancer. Ann Oncol 26: 141-148, 2015. PMID: 25316259. DOI: 10.1093/ annonc/mdu 472

7 Veer E Ter, Mohammad NH, Van Valkenhoef G, Ngai LL, Mali RMA, Anderegg MC, Van Oijen MGH and Van Laarhoven HWM: The efficacy and safety of first-line chemotherapy in advanced esophagogastric cancer: A network meta-analysis. J Natl Cancer Inst 108: 1-13, 2016. PMID: 27576566. DOI: 10.1093/jnci/djw 166

8 Hironaka S, Sugimoto N, Yamaguchi K, Moriwaki T, Komatsu Y, Nishina T, Tsuji A, Nakajima TE, Gotoh M, Machida N, Bando H, Esaki T, Emi Y, Sekikawa T, Matsumoto S, Takeuchi M, Boku N, Baba H and Hyodo I: S-1 plus leucovorin versus S1 plus leucovorin and oxaliplatin versus $\mathrm{S}-1$ plus cisplatin in patients with advanced gastric cancer: a randomised, multicentre, open-label, phase 2 trial. Lancet Oncol 17: 99-108, 2016. PMID: 26640036. DOI: 10.1016/S1470-2045(15)00410-6

9 Bleiberg H, Conroy T, Paillot B, Lacave AJ, Blijham G, Jacob JH, Bedenne L, Namer M, De Besi P, Gay F, Collette L and Sahmoud T: Randomised phase II study of cisplatin and 5fluorouracil (5-FU) versus cisplatin alone in advanced squamous cell oesophageal cancer. Eur J Cancer 33: 1216-1220, 1997. PMID: 9301445.

10 Iizuka T, Kakegawa T, Ide H, Ando N, Watanabe H, Tanaka O, Takagi I, Isono K, Ishida K and Arimori M: Phase II evaluation of cisplatin and 5-fluorouracil in advanced squamous cell carcinoma of the esophagus: a Japanese Esophageal Oncology Group Trial. Jpn J Clin Oncol 22: 172-176, 1992. PMID: 1518165.

11 Honda M, Miura A, Izumi Y, Kato T, Ryotokuji T, Monma K, Fujiwara J, Egashira H and Nemoto T: Doxorubicin, cisplatin, and fluorouracil combination therapy for metastatic esophageal squamous cell carcinoma. Dis Esophagus 23: 641-645, 2010. PMID: 20545978. DOI: 10.1111/j.1442-2050.2010.01070.x

12 Hironaka S, Tsubosa Y, Mizusawa J, Kii T, Kato K, Tsushima T, Chin K, Tomori A, Okuno T, Taniki T, Ura T, Matsushita H, Kojima T, Doki Y, Kusaba H, Fujitani K, Taira K, Seki S, Nakamura T, Kitagawa Y and Japan Esophageal Oncology Group/Japan Clinical Oncology Group: Phase I/II trial of 2weekly docetaxel combined with cisplatin plus fluorouracil in metastatic esophageal cancer (JCOG0807). Cancer Sci 105: 1189-1195, 2014. PMID: 25041052. DOI: 10.1111/cas.12486

13 Van Cutsem E, Moiseyenko VM, Tjulandin S, Majlis A, Constenla M, Boni C, Rodrigues A, Fodor M, Chao Y, Voznyi E, Risse ML, Ajani JA and V325 Study Group: Phase III study of docetaxel and cisplatin plus fluorouracil compared with cisplatin and fluorouracil as first-line therapy for advanced gastric cancer: a report of the V325 study group. J Clin Oncol 24: 4991-4997, 2006. PMID: 17075117. DOI: $10.1200 / J C O .2006 .06 .8429$

14 Shirakawa T, Kato K, Nagashima K, Nishikawa A, Sawada R, Takahashi N, Shoji H, Sasaki Y, Honma Y, Iwasa S, Takashima A, Okita N, Hamaguchi T, Yamada Y and Shimada Y: A retrospective study of docetaxel or paclitaxel in patients with advanced or recurrent esophageal squamous cell carcinoma who previously received fluoropyrimidine- and platinum-based chemotherapy. Cancer Chemother Pharmacol 74: 1207-1215, 2014. PMID: 25267597. DOI: 10.1007/s00280-014-2597-3

15 Kato K, Tahara M, Hironaka S, Muro K, Takiuchi H, Hamamoto Y, Imamoto $\mathrm{H}$, Amano $\mathrm{N}$ and Seriu $\mathrm{T}$ : A phase II study of paclitaxel by weekly 1-h infusion for advanced or recurrent esophageal cancer in patients who had previously received platinum-based chemotherapy. Cancer Chemother Pharmacol 67: 1265-1272, 2011. PMID: 20703479. DOI: 10.1007/s00280-010-1422-x

16 ter Veer E, Haj Mohammad N, van Valkenhoef G, Ngai LL, Mali RMA, van Oijen MGH and van Laarhoven HWM: Second- and third-line systemic therapy in patients with advanced esophagogastric cancer: a systematic review of the literature. Cancer Metastasis Rev 35: 439-456, 2016. PMID: 27417221. DOI: $10.1007 / \mathrm{s} 10555-016-9632-2$

17 Anandavadivelan P and Lagergren P: Cachexia in patients with oesophageal cancer. Nat Rev Clin Oncol 13: 185-198, 2016. PMID: 26573424. DOI: 10.1038/nrclinonc.2015.200

18 Cunningham D, Starling N, Rao S, Iveson T, Nicolson M, Coxon F, Middleton G, Daniel F, Oates J and Norman AR: Capecitabine and oxaliplatin for advanced esophagogastric cancer. N Engl J Med 358: 36-46, 2008. PMID: 18172173. DOI: 10.1056/ NEJMoa073149

19 Al-Batran S-E, Hartmann JT, Probst S, Schmalenberg H, Hollerbach S, Hofheinz R, Rethwisch V, Seipelt G, Homann N, Wilhelm G, Schuch G, Stoehlmacher J, Derigs HG, HegewischBecker S, Grossmann J, Pauligk C, Atmaca A, Bokemeyer C, Knuth A, Jäger $\mathrm{E}$ and Arbeitsgemeinschaft Internistische Onkologie: Phase III trial in metastatic gastroesophageal adenocarcinoma with fluorouracil, leucovorin plus either oxaliplatin or cisplatin: A study of the Arbeitsgemeinschaft Internistische Onkologie. J Clin Oncol 26: 1435-1442, 2008. PMID: 18349393. DOI: 10.1200/JCO.2007.13.9378

20 Van Meerten E, Eskens FALM, Van Gameren EC, Doorn L and Van Der Gaast A: First-line treatment with oxaliplatin and capecitabine in patients with advanced or metastatic oesophageal cancer: A phase II study. Br J Cancer 96: 1348-1352, 2007. PMID: 17437008. DOI: 10.1038/sj.bjc.6603750

21 Rixe O, Ortuzar W, Alvarez M, Parker R, Reed E, Paull K and Fojo T: Oxaliplatin, tetraplatin, cisplatin, and carboplatin: spectrum of activity in drug-resistant cell lines and in the cell lines of the National Cancer Institute's Anticancer Drug Screen panel. Biochem Pharmacol 52: 1855-1865, 1996. PMID: 8951344.

22 Kim DY, Kim JH, Lee SH, Kim TY, Heo DS, Bang YJ and Kim NK: Phase II study of oxaliplatin, 5-fluorouracil and leucovorin in previously platinum-treated patients with advanced gastric cancer. Ann Oncol 14: 383-387, 2003. PMID: 31321276. DOI: 10.1093/annonc/mdg106

23 Conroy T, Galais MP, Raoul JL, Bouché O, Gourgou-Bourgade S, Douillard JY, Etienne PL, Boige V, Martel-Lafay I, Michel P, Llacer-Moscardo C, François E, Créhange G, Abdelghani M Ben, Juzyna B, Bedenne L and Adenis A: Definitive chemoradiotherapy with FOLFOX versus fluorouracil and cisplatin in patients with oesophageal cancer (PRODIGE5/ ACCORD17): Final results of a randomised, phase $2 / 3$ trial. Lancet Oncol 15: 305-314, 2014. PMID: 24556041. DOI: 10.1016/S1470-2045(14)70028-2

24 Wang J, Chang J, Yu H, Wu X, Wang H, Li W, Ji D and Peng W: A phase II study of oxaliplatin in combination with leucovorin and fluorouracil as first-line chemotherapy in patients with metastatic squamous cell carcinoma of esophagus. Cancer Chemother Pharmacol 71: 905-911, 2013. PMID: 23370661. DOI: $10.1007 / \mathrm{s} 00280-013-2081-5$

Received June 9, 2019

Revised July 26, 2019

Accepted August 1, 2019 